\title{
Commentary
}

\section{Remember that? Or Does it just Seem Familiar? A Sophisticated Test for Assessing Memory in Humans and Animals Reveals a Specific Cognitive Impairment Following General Anesthesia in Infancy}

\author{
Howard Eichenbaum*,' \\ 'Department of Psychology, Center for Memory and Brain, Boston University, Boston, MA, USA
}

Neuropsychopharmacology (2014) 39, 2273-2274; doi:I0.I038/npp.2014.I I6

A decade ago, controversy raged over whether animals provided a valid model of the memory loss that is observed in humans following damage to the hippocampus. Whereas human amnesic patients are typically quite deficient in tests of recognition for recently seen words or pictures, monkeys and rats with selective hippocampal damage were hardly impaired in a standard recognition test where identified novel objects against familiar ones (Eichenbaum et al, 2007). However, this puzzle was resolved when Fortin et al (2004) used a sophisticated test that distinguished two cognitive processes that can support recognition in animals. The test had a history in studies of human memory that distinguished the ability to recollect a previous experience with a stimulus or simply feel that the stimulus is familiar as a result of exposure-think of the last time you saw a familiar face on the street, but could not remember who he was or why you knew him. Fortin et al applied this test to rats and found that, although overall recognition performance was not impaired after hippocampal damage, recollection was severely affected while familiarity was spared. Thus, a test that separates these two cognitive processes aligned the human and animal performance capacities. This approach has proven useful in detecting selective recollection impairments in humans with hippocampal pathology and mild cognitive impairment (Aggleton et al, 2005; Ally et al, 2009). Now, Stratmann et al have successfully used this test to reveal a selective recollection impairment following general anesthesia in infancy in humans and in a rodent model. Notably, the current literature is mixed on whether there are negative effects of general anesthesia in infancy, so a sensitive measure will surely be required to detect and characterize any

*Correspondence: Dr H Eichenbaum, Department of Psychology, Boston University, 64 Cummington St, Boston, MA 02215, USA, Tel: + I $410617 \quad 353$ |426, Fax: +I $410 \quad 617 \quad 353$ 14l4, E-mail: hbe@bu.edu

Received I3 May 2014; accepted I3 May 2014 impairment, and a test that separates components of memory may be just what is needed.

How does one separate recollection from familiarity in animals? The method employs signal detection theory to calculate a receiver operating curve (ROC) for differentiating items in a studied list from new items along with varying the subject's bias towards 'old' or 'new' responses. In the typical test for humans, subjects initially study a list of words, then are presented with words from the list (old items) and new words in mixed order, and finally judge their level of confidence (that is, bias) on a scale. The ROC is the function that the ratio of correct identifications of old words (hits) against erroneous identifications of new words as old (false alarms) over the series of bias points. Healthy young humans exhibit an ROC function that is curvilinear and asymmetrical (Figure 1a). The curvilinear component is thought to reflect the strength of familiarity driving detection of old items (' $\mathrm{F}$ ' in Figure 1a), whereas the asymmetry is thought to reflect improved detection of old items based on additional memory for associations (eg, it was the first one on the list, or it was next to another particular item), which is the hallmark of recollection (' $R$ ' in Figure 1a). The validity of these interpretations is evident in dissociations of the two ROC components. When the list items are pairs of associated words and new items are mispaired words from the study list, the ROC remains asymmetrical and becomes linear, reflecting the inability to distinguish items based on familiarity of the word elements in each pairing (Figure 1b). Conversely, when recognition judgments are rushed, the ROC instead remains curvilinear, but becomes symmetrical, reflecting the difference between a fast familiarity process and the elimination of slower occurring recollection (Figure 1c; Eichenbaum et al, 2010). What is so marvelous about the ROC approach is that one can separately measure the capacities for recollection and familiarity without asking subjects what they remember. The protocol simply requires them to study a list of stimuli, then provide differential responses to 'old' and 'new' items across a series of bias levels for 'new' or 'old' responses-humans and animals 

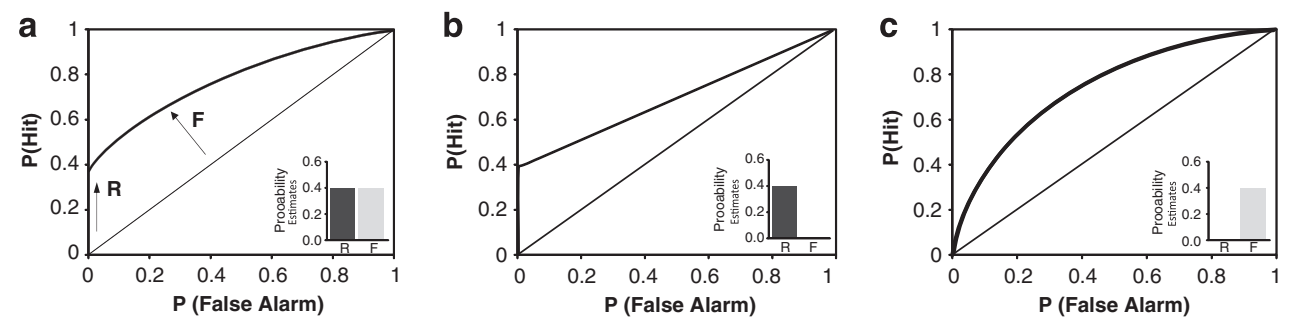

Figure I ROC functions in (a) the standard test of item recognition, (b) associative recognition, and (c) item recognition with a response deadline. Inserts compare index measures of the recollection (R) and familiarity $(F)$ components of the ROC function. ROC, receiver operating curve.

alike can be trained to distinguish old and new stimuli and, across species, their response biases are influenced by task parameters.

The Stratmann et al paper used the standard version of the ROC protocol on humans and an adaptation of the task for rodents that had been developed by Fortin et al (2004). In the animal version, the list stimuli were common household scents (eg, oregano, cinnamon) and, instead of separating response biases by confidence judgments, Fortin et al varied the reward payoff ratio across blocks of trials to induce response biases. This method reproduces in rats the same ROC functions as observed in humans (Figure 1). Stratmann et al applied this approach to measure the effects of general anesthesia in infancy in both humans and in a rat model, and reported parallel dissociations in both species. General anesthesia in infancy results in a selective impairment in recollection, sparing familiarity, in both humans and rats. The success of the rodent model in this study was important, because general anesthesia is used only concurrent with a medical condition in humans, thus confounding the effects of anesthesia with the source of the medical condition or the effects of the surgery itself. Here, the rodent model was used on healthy animals and a surgical control was added. These findings have considerable significance for the use of general anesthesia in infancy.

Tests of memory in animal models are widely used to assess cognitive impairment as well as the efficacy of treatments that could improve cognition. Yet, nearly all animal tests involve protocols very unlike those used in humans (eg, the water maze, contextual fear conditioning) or recognition protocols that do not separate recollection and familiarity. The ROC approach requires more sophisticated and prolonged testing than the other more commonly used tests, but has proven quite fruitful for detecting specific impairments in cognition associated with aging, anesthesia, and in differentiating the effects of prefrontal versus hippocampal pathology (Eichenbaum et al, 2010).

\section{FUNDING AND DISCLOSURE}

Dr Eichenbaum is supported by grants from NIMH (MH51570, MH094263). The author declared no conflict of interest.

\section{REFERENCES}

Aggleton J, Vann S, Denby C, Dix S, Mayes A, Roberts N et al (2005). Sparing of the familiarity component of recognition memory in a patient with hippocampal pathology. Neuropsychologia 43: 1810-1823.

Ally BA, Gold CA, Budson AE (2009). An evaluation of recollection and familiarity in Alzheimer's disease and mild cognitive impairment using receiver operatinig characteristics. Brain Cogn 69: $504-513$.

Eichenbaum H, Yonelinas AR, Ranganath C (2007). The medial temporal lobe and recognition memory. Ann Rev Neurosci 30: 123-152.

Eichenbaum H, Fortin N, Sauvage M, Robitsek RJ, Farovik A (2010). An animal model of amnesia that uses receiver operating characteristics (ROC) analysis to distinguish recollection from familiarity deficits in recognition memory. Neuropsychologia 48: 2281-2289.

Fortin NJ, Wright SP, Eichenbaum H (2004). Recollection-like memory retrieval in rats is dependent on the hippocampus. Nature 431: 188-191.

Stratmann G, Lee J, Sall JW, Lee BH, Alvi RS, Shih J et al (2014). Effect of general anesthesia in infancy on long-term recognition memory in humans and rats. Neuropsychopharmacology 39: $2275-2287$. 